\title{
Decline in the prevalence HIV among pregnant women attending antenatal clinics in Tanzania, 2001-2011
}

\author{
JOEL MANYAHI'* BONIPHACE S. JULLU², MATHIAS I. ABUYA ${ }^{3}$, JAMES JUMA ${ }^{3}$, BONITA KILAMA ${ }^{3}$, VERYEH \\ SAMBU ${ }^{3}$, JOSEF NONDI 3 , BERNARD RABIEL 3 , NEEMA MAKYAO ${ }^{3}$, ANGELA RAMADHANI ${ }^{3}$, GEOFFREY SOMI 3 \\ and MECKY I. MATEE \\ ${ }^{1}$ Muhimbili University of Health and Allied Sciences, P.O. Box 65001, Dar es Salaam, Tanzania \\ ${ }^{2}$ St. Francis University College of Health and Allied Sciences, P.O. Box 175, Ifakara, Tanzania \\ ${ }^{3}$ Ministry of Health, Community Development, Gender, Elderly and Children, National AIDS Control Programme, \\ Dar es Salaam, Tanzania
}

\begin{abstract}
Background: The Tanzania National AIDS Control Programme has established HIV sentinel surveillance among antenatal clinic (ANC) attendees as one of the methods for collecting data on HIV prevalence. This article provides trends on HIV prevalence for 92 sentinel sites that have constantly been part of the surveillance system since 2001 and have participated in at least three consecutive rounds.

Method: The surveillance population included all pregnant women aged 15-49 years who were attending a selected sentinel ANC site for the first time for any pregnancy between 2001 and 2011. Serial testing for HIV infection was done anonymously by detecting for the presence of IgG antibodies to HIV on dried blood spot (DBS) specimens. HIV trends were calculated taking into account random effects from sites on the following variables: region, sites and socio-demographic characteristics defined as age, marital status, parity, education level and duration of stay at present residence.

Results: Overall, there was a significant decline in HIV prevalence from 9.6\% in 2001 to $5.6 \%$ in 2011 ( $p<0.01$ ). Specifically, the HIV prevalence among 15-24 years' pregnant women significantly declined from $7.8 \%$ in $2001 / 2002$ to $4 \%$ in 2011 ( $p<0.01$ ). The decline in HIV prevalence occurred irrespective of residence, marital status, education level or previous pregnancies.

Conclusion: There has been a significant decline in HIV infections among young pregnant women attending ANC clinics in Tanzania since 2001. This study also indicates that ANC surveillance among pregnant women over time can provide useful estimates of HIV situation between the population surveys.
\end{abstract}

Keywords: HIV/AIDS, prevalence, surveillance, pregnant women, antenatal care, Tanzania

\section{Introduction}

Since 1990, the Tanzanian National AIDS Control Programme (NACP) has established human immunodeficiency virus (HIV) sentinel surveillance system among antenatal clinic (ANC) attendees (Kwesigabo et al., 2000). The aim is to monitor the trend in the prevalence of HIV infection over time as well as provide evidence-based data to for public health decision making, including resource allocation, monitoring and evaluating prevention and care programmes. Between 1990 and 1998, data were being collected bi-annually. However, since 1998 data are collected annually. At the beginning, HIV testing was being done on the whole blood samples collected largely from sentinel sites located in urban areas and tested in respective regional hospitals due to cold chain challenges in the transportation of the samples. The introduction of the dried blood spots (DBS) technique in 2000 (Cassol et al., 1997) simplified handling and transportation of freshly collected samples, contributing to the scale up to more ANC surveillance sites, including those located in remote and hard to reach areas.

Tanzania adopted ANC HIV surveillance procedures based on UNAIDS/WHO guidelines (UNAIDS/WHO, 1996). Between 2001 and 2011, NACP has conducted five rounds of ANC HIV surveillance (MoHSW, 2014). The numbers of ANC sentinel sites and Regional coverage as well as geographical coverage have steadily increased from 24 (in six Regions) during 2000/2001 (largely urban), to 133 (urban, semi-urban and rural) in all 21 regions of mainland Tanzania by 2011. Data

\footnotetext{
*Correspondence E-mail: manyahijoel@yahoo.com
} 
from three population-based surveillances among adults aged 15-49 years in Tanzania have revealed a decrease in the magnitude of HIV infection with variations by regions (THIS, 2005; THMIS, 2008, 2012). In both surveys HIV prevalence was higher in women than men. However, ANC based sentinel surveillance has been limited to women and continues to be an instrumental data source for estimating HIV prevalence trends over time. Consequently, HIV prevalence trends data at national, regional and sub-group levels are critical for providing the necessary information for proper monitoring, evaluation and programming as well as mobilization of political commitment. This article analysed trends in the HIV prevalence in 92 sentinel sites in Tanzania that have participated in at least three consecutive ANC HIV surveillance rounds from 2001 to 2011.

\section{Materials and Methods}

\section{Surveillance target population}

The surveillance population included all pregnant women aged 15-49 years who were attending a selected sentinel ANC site for the first time at any pregnancy between 2001 and 2011. Surveillance staff recorded social and demographic characteristics of the women that included age, marital status, parity, educational level, and duration of stay at present residence. The data collection periods for all ANC surveillances rounds were three months.

\section{Specimen collection}

In 2001, ANC surveillance HIV testing was done on whole blood samples and testing were done in respective regional hospitals. From 2003-04 to 2011 ANC surveillance, a drop (100 $\mu$ l) of leftover whole blood from syphilis testing was used to prepare dried blood spot (DBS) specimens for HIV surveillance purposes. The procedure for DBS preparation and transport has been explained elsewhere (Manyahi et al., 2015). The DBS cards were labelled matching to surveillance data collection form. At this point, the upper part of the surveillance data collection form that contained the woman's clinic card number was torn up and discarded in order to ensure delinking of the client identifier from the respective HIV data.

Dried DBS cards were stacked between weighing paper and stored in zip-locked plastic bags with desiccant (drying) packets and a humidity indicator card together with their completed data collection forms. Desiccant packs were changed when humidity indicator cards changed colour from blue to pink. On weekly basis using courier service, ANC survey staff mailed completed data collection forms and DBS samples to their assigned zonal laboratory. Upon receipt of the samples at the zonal laboratory, the surveillance numbers for each sample were recorded in a logbook. The NACP regularly monitored the volume of DBS received by zonal centres from each ANC and contacted the clinics which had posted unlinked numbers for reconciliation. Then all DBS specimens collected were sent to the Department of Microbiology and Immunology at Muhimbili University of Health and Allied Sciences for testing. Testing for the presence of IgG antibodies to HIV and quality assurance were performed according to a previously described protocol (Manyahi et al., 2015).

\section{Data analysis}

Data analysis was carried out using Stata v.12 software (Stata Corporation, College Station, Texas, USA). Trends of HIV point prevalence were done; $p$-trends were calculated taking into account random effects from sites. The $X^{2}$ test and tests for linear trend were used as appropriate; $p$-value of less than 0.05 was considered statistically significant.

\section{Ethical considerations}

Because syphilis screening is already routinely conducted and HIV testing was performed on nonlinked samples, informed consent was not warranted. Collected data was de-identified before 
analysis to protect client confidentiality. The surveillance protocols received ethical approval from the Medical Research Coordinating Committee of the National Institute for Medical Research.

\section{Results}

Results show a significant decline in HIV prevalence from $9.6 \%(95 \% \mathrm{Cl}=8.9,10.2)$ in $2001 / 2002$ to $5.6 \%$ (95\% C.I 5.4, 5.8) in 2011 ( $\mathrm{p}<0.01$ ) (Figure 1). Likewise, HIV prevalence among pregnant young women aged 15-24 years has decreased substantially in urban (from $8.8 \%$ to $4.0 \%$ ), semi-urban (from $8.5 \%$ to $4.0 \%$ ) and rural settings (from 3\% to $2.2 \%$ ) (Figure 2 ).

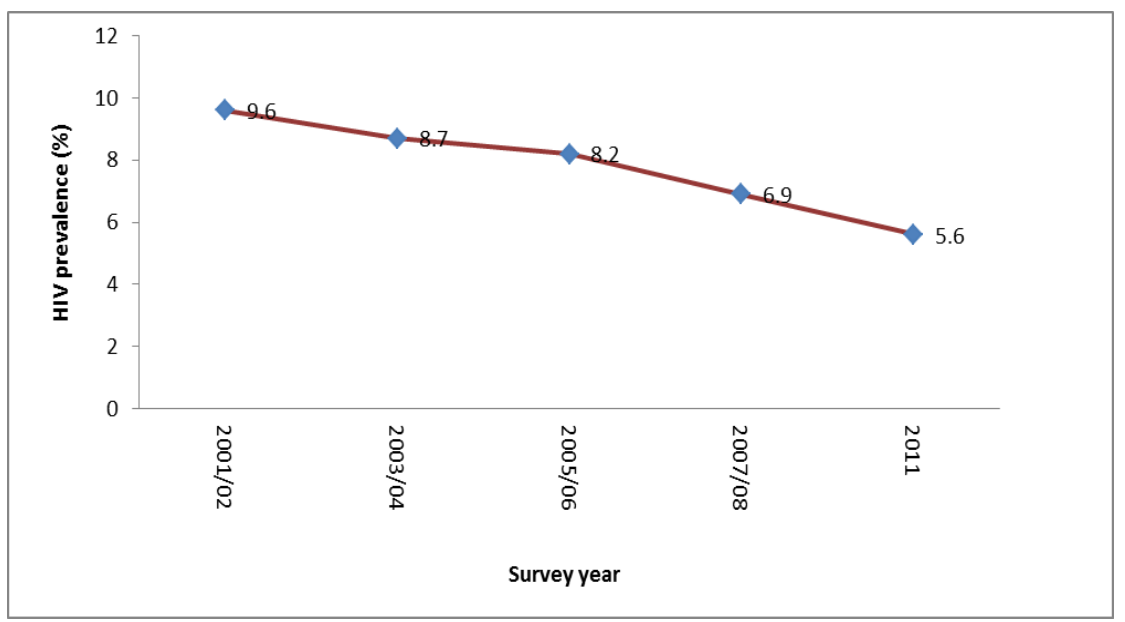

Figure 1: Trends of HIV prevalence among ANC attendees (2001-2011)

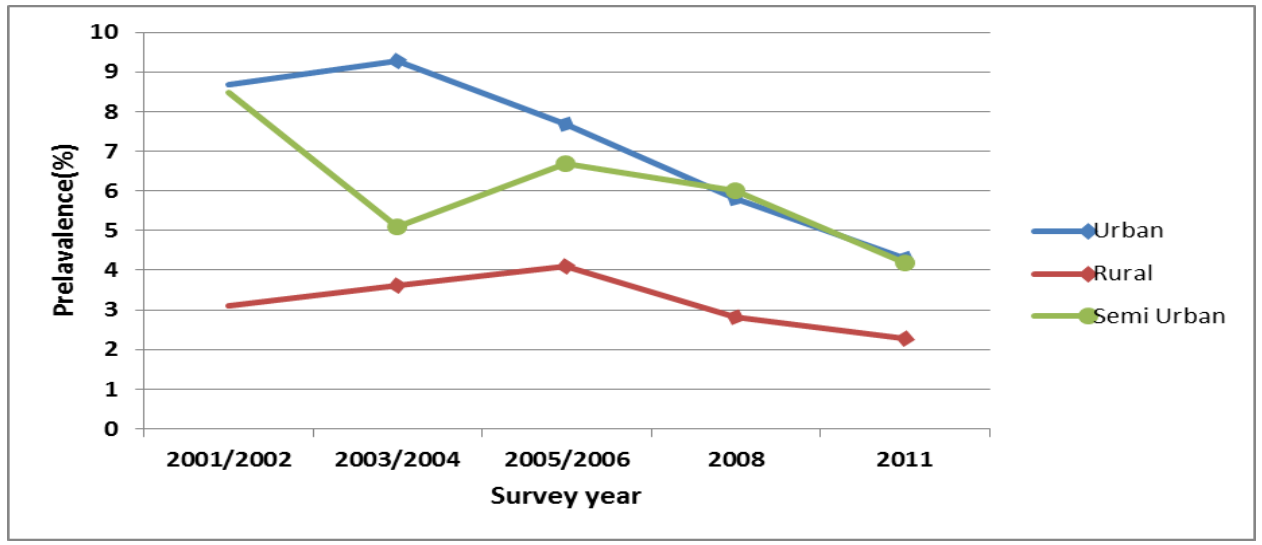

Figure 2 : HIV prevalence trends among ANC attendees aged 15-24 years by residence

Point estimates calculated from the 24 sites that had five data points, showed HIV prevalence declined significantly from $9.7 \%(95 \% \mathrm{Cl}=8.9,10.2)$ in $2001 / 2002$, to $5.8(95 \% \mathrm{Cl}=5.3,6.3)$ in 2011 $(p=0.0001)$ (Figure 3 ).

Decreasing HIV prevalence trends were significant in all age groups except $>35$ years and older from 2001 to 2011 (Table 1). Further decreases $(p<0.001)$ were seen in both single and married pregnant women (from $12.6 \%$ to $6.8 \%$ and from $9.1 \%$ to $5.4 \%$, respectively,), and urban (from $12.1 \%$ to $6.6 \%$ ) and rural (from $4.1 \%$ to $3.1 \%$, p <0.001) pregnant women. The decline in HIV prevalence occurred irrespective of the number of previous pregnancies or education status, $(p<0.001)$. 


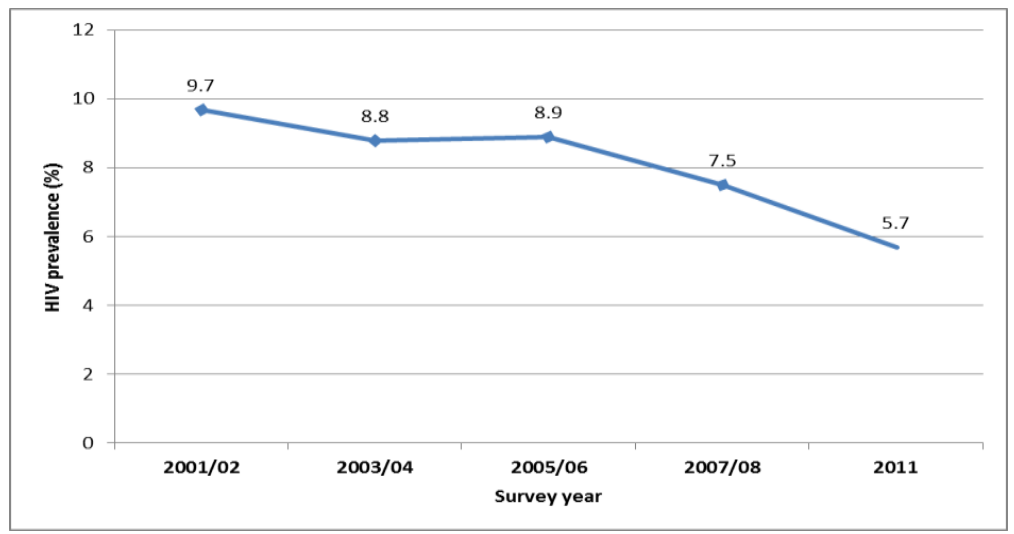

Figure 3: Trend of HIV prevalence among ANC attendees of all ages

Table 1: HIV prevalence trends by social-demographic characteristics 2001- 2011

\begin{tabular}{|c|c|c|c|c|c|c|c|}
\hline \multirow[t]{2}{*}{ Variable } & \multirow[t]{2}{*}{ Response } & \multicolumn{5}{|c|}{ Prevalence } & \multirow[t]{2}{*}{ P-value } \\
\hline & & $2001 / 02$ & $2003 / 4$ & $2005 / 06$ & $2007 / 08$ & 2011 & \\
\hline \multirow[t]{3}{*}{ Age (years) } & $15-24$ & 7.6 & 7.4 & 6.8 & $5 \cdot 3$ & 3.9 & $<0.001$ \\
\hline & $25-34$ & 13 & 11 & 9.9 & 8.9 & 7.3 & $<0.001$ \\
\hline & $35+$ & 7 & 6.7 & 8.1 & 7.2 & 7 & 0.984 \\
\hline \multirow[t]{3}{*}{ Education } & None & 6.4 & 5.2 & 5.5 & 4.8 & 4.5 & 0.012 \\
\hline & Primary & 10.2 & $9 \cdot 3$ & 8.6 & 7.5 & 6 & $<0.001$ \\
\hline & Secondary+ & & & $9 \cdot 3$ & 7 & 4.9 & $<0.001$ \\
\hline \multirow[t]{4}{*}{ Marital status } & Single & 12.6 & 9.7 & 8.9 & 7 & 6.8 & $<0.001$ \\
\hline & Married & 9.1 & 8.6 & 8.1 & 6.9 & 5.4 & $<0.001$ \\
\hline & Divorced & - & - & - & - & 5.1 & \\
\hline & Others & - & - & - & 13 & 8.7 & \\
\hline \multirow[t]{3}{*}{ Residence } & Urban & 12.1 & 11.2 & $9 \cdot 9$ & 8.4 & 6.6 & $<0.001$ \\
\hline & Semi-urban & 3.7 & 4.7 & 7.8 & 7.5 & 5.8 & 0.010 \\
\hline & Rural & 4.1 & 3.7 & 4.4 & 3.4 & 3.1 & $<0.001$ \\
\hline \multirow[t]{4}{*}{ Previous pregnancy } & 0 & 7.2 & 7 & 6.5 & 4.7 & 3.5 & $<0.001$ \\
\hline & $1-2$ & 11 & 10.2 & 9.3 & 8.2 & 6.5 & $<0.001$ \\
\hline & $3-4$ & 11.6 & 9.4 & 9.1 & 7.6 & $7 \cdot 3$ & $<0.001$ \\
\hline & $5^{+}$ & 6.5 & 5.4 & 5.4 & 5.4 & 4.2 & $<0.001$ \\
\hline
\end{tabular}

At regional level, the most consistent and significant HIV prevalence declines were observed among pregnant women in Dar es Salaam (from 12.8\% to 6.08\%), Dodoma (6.1\% to 1.96 ), Mbeya (16.0\% to $11.31 \%$ ), Kigoma (from 5.1\% to $1.31 \%$ ), Tanga (from 9.2\% to $4.76 \%$ ), and Arusha (from $6.1 \%$ to $2.84 \%$ ) $\mathrm{p}<0.001$ (Table 2). Furthermore, substantial HIV prevalence declines were observed among ANC attendees in Kilimanjaro, Morogoro, Mara, Tabora and Iringa regions. Notably, Kagera and Mtwara regions which have participated in five ANC rounds showed insignificant changes in HIV prevalence trends $(p=0.2)$.

When the results of ANC surveillance were compared with those of population-based surveys, an overestimation of HIV prevalence in ANC survey in early rounds (2002-2008) was observed. However, in the consequent years, the prevalence between ANC and population-based surveys were similar (Figure 4). 


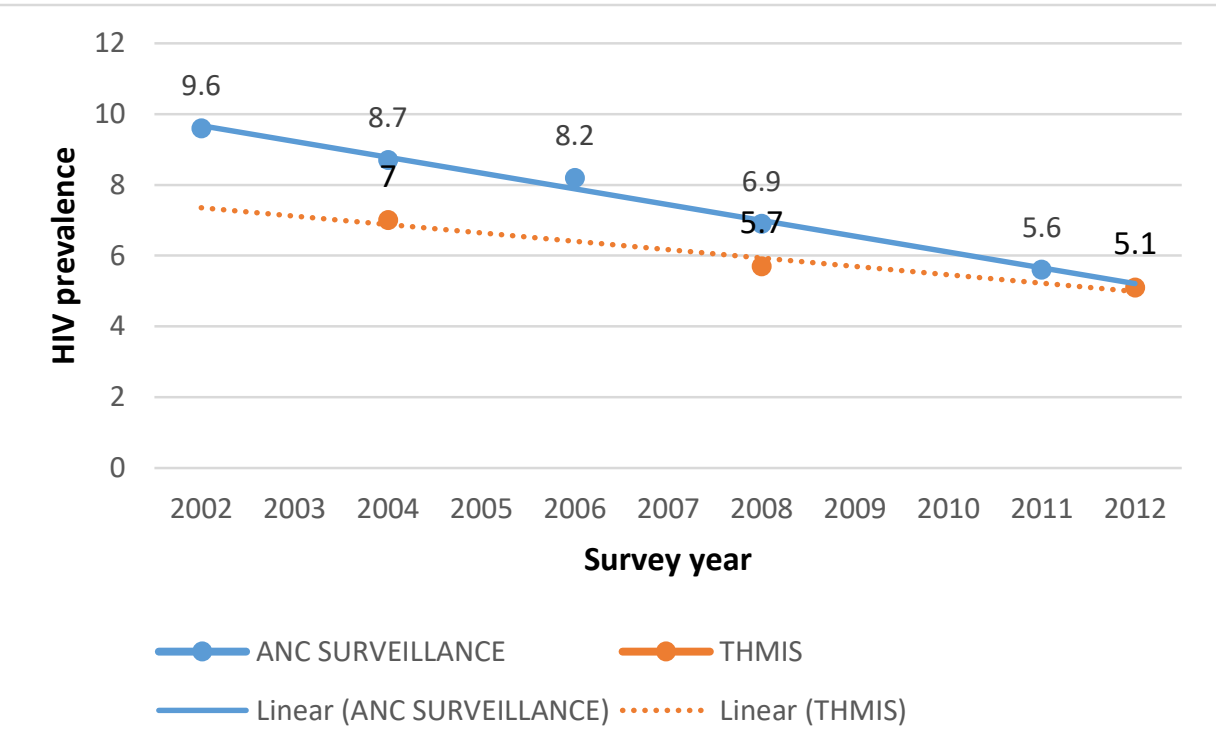

Figure 4: Comparison of HIV trends between data from ANC surveillance and population surveys

Table 2: Trends of HIV prevalence among ANC attendees by region $2001-2011$

\begin{tabular}{lllllll}
\hline Region & $\begin{array}{l}\text { Prevalence } \\
\text { 2001/02 }\end{array}$ & $\mathbf{2 0 0 3 / 0 4}$ & $\mathbf{2 0 0 5 / 0 6}$ & $\mathbf{2 0 0 7 / 0 8}$ & $\mathbf{2 0 1 1}$ & P-value \\
\hline Dar es Salaam & 12.8 & 10.8 & 10.9 & 7 & 6.1 & $<0.001$ \\
Dodoma & 6.1 & 7.8 & 6.1 & 5.4 & 2 & $<0.001$ \\
Kagera & 5.6 & 4.7 & 4.7 & 4.8 & 4.6 & 0.238 \\
Kilimanjaro & 6.3 & 5.7 & 5 & 4.57 & 3.2 & 0.001 \\
Mbeya & 16 & 15.7 & 15.9 & 12.6 & 11.3 & $<0.001$ \\
Mtwara & 7.1 & 5.1 & 6.1 & 6.8 & 5.5 & 0.216 \\
Morogoro & - & 9 & 7.2 & 6.59 & 5.6 & 0.001 \\
Kigoma & - & 5.1 & 3.5 & 1.53 & 1.3 & $<0.001$ \\
Lindi & - & 7.1 & 9.4 & 5.7 & 5.4 & 0.137 \\
Tanga & - & 9.2 & 6.5 & 5.89 & 4.8 & $<0.001$ \\
Arusha & - & - & 6.1 & 5.6 & 2.8 & $<0.001$ \\
Mara & - & - & 6.1 & 4.4 & 4.2 & 0.014 \\
Iringa & - & - & 18.2 & 16.5 & 14.8 & 0.044 \\
Shinyanga & - & - & 6.8 & 7.1 & 5.8 & 0.133 \\
Tabora & - & - & 7.2 & 6.5 & 4.7 & 0.004 \\
\hline
\end{tabular}

\section{Discussion}

This study shows a significant decline in HIV prevalence among pregnant women attending ANC in Tanzania from 2001 to 2011. The finding is in agreement with those from population surveys in Tanzania (THIS 2005, THMIS 2008, 2012), which have shown steady decline in HIV prevalence from 7\% in 2004, 5.7\% in 2008 and 5.1\% in 2011. In Tanzania HIV/AIDS and Malaria Indicator Surveys, HIV prevalence among women declined from $7.7 \%$ in 2004, to $6.6 \%$ in 2008 and $6.2 \%$ in 2011 (THIS 2005; THMIS 2008, 2012). Comparing results of ANC surveillance with those of THMIS, we find an overestimation of HIV prevalence in ANC survey in early rounds, however later ANC and THMIS showed similar prevalence. Most likely, the increase in the number of sites and geographical coverage with each round has increased representativeness of the ANC survey. In addition, there has been a change in the nature of the HIV epidemic from concentrated to a more generalized form (THMS 2012). 
The decline in HIV infection occurred irrespective of residential, marital status, education level and previous pregnancies. These findings are in agreement with results of population based surveys (THMS 2008, 2012) and other studies in Tanzania (Wambura et al., 2007; Hargreaves \& Howe, 2010; Kumogola et al., 2010). A significant reduction in HIV prevalence was also observed among young pregnant women aged 15-24 years, which signifies a reduction in new HIV infections (Zaba et al., 2000; Ghys et al., 2010). The 2011/2012 population survey in Tanzania have documented an increase in the age of sexual debut, male circumcision, consistent condom utilization, coverage rates HIV testing and counselling services, as well as in availability and access to antiretroviral treatment (THMIS, 2012). Similarly, others studies in Tanzania have reported significant change in sexual risk behaviours (Lugalla et al., 2004; Mmbaga et al., 2007). We also register positive impact resulted from different intervention activities by both the Government and development partners; collectively, these measures could explain the observed decline in HIV prevalence.

Despite the general decline in HIV infection among the ANC attendees we found two exceptions. First, there were four regions (Kagera, Mtwara, Lindi, Shinyanga) with no significant decline trend in HIV prevalence. Kagera is a region where HIV/AIDS epidemic was first reported in Tanzania in 1983 (Kwesigabo et al., 2000) and the epidemic in that region matured, stabilized and declined earlier compared to other regions (Kwesigabo et al., 1998, 2000, 2005). The trend in the other three regions could be attributed to a number of factors such as socio-cultural, knowledge, attitudes and practices, which however were not investigated in these sentinel surveys. Secondly, we observed that over the years HIV prevalence among women aged above 35 years has stabilized at around $7 \%$. This finding could partly be attributed to an aging cohort effect (cumulative HIV infection case load). People including women, are living longer with the disease as result of substantial availability of anti-retrovirus treatment in reducing HIV associated morbidity and mortality (Quinn, 2008).

One of limitations of our study was change in blood collection procedures and testing facilities. The first surveillance testing was done at regional level facilities using whole blood while the rest were done at Muhimbili University of Health and Allied Sciences, and DBS were used. Moreover, although ANC surveillance data remains a useful tool to monitor HIV epidemic it is still an estimate of the HIV prevalence in the general population.

In conclusion, there is a significant decline in HIV infections among young pregnant women attending ANC clinics in Tanzania since 2001. This study indicates ANC surveillance among pregnant women over time can provide useful estimates of HIV situation between the population surveys.

\section{Competing interests}

The authors declare that they have no competing interests.

\section{Authors' contributions}

Authors contributed equally to this study. All authors read and approved the final manuscript.

\section{Acknowledgements}

This work was a collaborative effort of Tanzania National AIDS Control Programme, Muhimbili University of Health and Allied Sciences and St. Francis University College of Health and Allied Sciences. We specifically wish to thank the US Centers for Disease Control and Prevention for their technical assistance. 


\section{References}

Cassol, S., Gill, M.J., Pilon, R., Cormier, M., Voigt, R.F., Willoughby, B. \& Forbes, J. (1997) Quantification of human immunodeficiency virus type 1 RNA from dried plasma spots collected on filter paper. Journal of Clinical Microbiology 35:2795-801.

Ghys, P.D., Gouws, E., Lyerla, R., Garcia-Calleja, J.M., Barrerre, B., Serrano, D., Velasquez, C., Deleveaux, C., Gill, W., Mmelesi. M., Niyongere, A., Mboui Bilon, E., Longo, Jde D., Massanga, M., Guidaoussou, D., Konan Diby, J.P., Kibangou, N., Woldu, A., Bikoma, F., Duval, N., Spring, K., Barsigo, A., Nkonyana, J., Wandonda, N., Jonas, A., Kawu, I., Mutagoma, M., Mosala, T., Mndzebele, S., Odido, H., Agbogan, Y.D., Musinguzi, J., Somi, G., Gboun, M., Phiri, D., Gonese, E., Gregson, S. \& Mugurungi, O. (2010) Trends in HIV prevalence and sexual behaviour among young people aged 15-24 years in countries most affected by HIV. Sexually Transmitted Infection 86 (Suppl 2): ii72-ii83).

Hargreaves, J.R \&, Howe, L.D. (2010) Changes in HIV prevalence among differently educated groups in Tanzania between 2003 and 2007. AIDS 24: 755-761.

Kumogola, Y., Slaymaker, E., Zaba, B., Mngara, J., Isingo, R., Changalucha, J., Mwidunda, P., Kimaro, D. \& Urassa, M. (2010) Trends in HIV \& syphilis prevalence and correlates of HIV infection: results from cross-sectional surveys among women attending ante-natal clinics in Northern Tanzania. BMC Public Health 10: 553.

Kwesigabo, G., Killewo, J., Godoy, C., Urassa, W., Mbena, E., Mhalu, F., Biberfeld, G., Wall, S. \& Sandstrom, A. (1998) Decline in the prevalence of HIV-1 infection in young women in the Kagera region of Tanzania. Journal of Acquired Immune Deficiency Syndrome and Hum Retrovirology 17:262-268.

Kwesigabo, G., Killewo, J., Urassa, W., Lugalla, J., Emmelin, M., Mutembei, A., Mhalu, F., Biberfeld, G., Wall, S. \& Sandstrom, A. (2005) HIV-1 infection prevalence and incidence trends in areas of contrasting levels of infection in the Kagera Region, Tanzania, 19872000. Journal of Acquired Immune Deficiency Syndromes 40: 585-591.

Kwesigabo, G., Killewo, J.Z., Urassa, W., Mbena, E., Mhalu, F., Lugalla, J.L., Godoy, C., Biberfeld, G., Emmelin, M., Wall, S. \& Sandstrom, A. (2000) Monitoring of HIV-1 infection prevalence and trends in the general population using pregnant women as a sentinel population: 9 years experience from the Kagera region of Tanzania. Journal of Acquired Immune Deficiency Syndrome 23: 410-417.

Lugalla, J., Emmelin, M., Mutembei, A., Sima, M., Kwesigabo, G., Killewo, J. \& Dahlgren, L. (2004) Social, cultural and sexual behavioral determinants of observed decline in HIV infection trends: lessons from the Kagera Region, Tanzania. Social Science \& Medicine 59: 185-198.

Manyahi, J., Jullu, B.S., Abuya, M.I., Juma, J., Ndayongeje, J., Kilama, B., Sambu, V., Nondi, J., Rabiel, B., Somi, G. \& Matee, M.I. (2015) Prevalence of HIV and syphilis infections among pregnant women attending antenatal clinics in Tanzania, 2011. BMC Public Health 15: 501.

Mmbaga, E., Hussain, A., Leyna, G., Holm-Hansen, C., Mnyika, K., Sam, N., Klouman, E. \& Klepp, K.I. (2007) Trends in HIV-1 prevalence and risk behaviours over 15 years in a rural population in Kilimanjaro region of Tanzania. AIDS Research and Therapy 4: 23.

MoHSW (2014) Surveillance of HIV and Syphilis Among Antenatal Clinic Attendees, 2011. National AIDS Control Programme, Ministry of Health and Social Welfare, The United Republic of Tanzania.

Quinn, TC (2008). HIV epidemiology and the effects of antiviral therapy on long-term consequences. AIDS 22(Suppl 3): S7-12

THIS (2005) Tanzania HIV/AIDS Indicator Survey 2003-04. Tanzania Commission for AIDS, National Bureau of Statistics and ORC Macro International Inc.

THMIS (2008) Tanzania HIV/AIDS and Malaria Indicator Survey 2007-08. Tanzania Commission for AIDS, Zanzibar AIDS Commission, National Bureau of Statistics, Office of the Chief Government Statistician and Macro International Inc. 
THMIS (2013) Tanzania HIV/AIDS and Malaria Indicator Survey 2011-12. Tanzania Commission for AIDS, Zanzibar AIDS Commission, National Bureau of Statistics, Office of the Chief Government Statistician and ICF International 2013.

UNAIDS/WHO (1996) Working Group on Global HIV/AIDS and STI Surveillance. Guidelines for conducting HIV sentinel sero-surveys among pregnant women and other groups. Geneva: World Health Organization.

Wambura, M., Urassa, M., Isingo, R. Ndege, M., Marston, M., Slaymaker, E., Mngara, J., Changalucha, J., Boerma, T.J. \& Zaba, B. (2007) HIV prevalence and incidence in rural Tanzania: results from 10 years of follow-up in an open-cohort study. Journal of Acquired Immune Deficiency Syndrome 46: 616-623.

Zaba, B., Boerma, T. \& White, R. (2000) Monitoring the AIDS epidemic using HIV prevalence data among young women attending antenatal clinics: prospects and problems. AIDS 14: 16331645 . 